\title{
Cartography on the Internet: Thoughts and a Preliminary User Survey
}

This paper reviews the design and delivery of maps through the Internet. The paper also reports on the findings of a pilot study undertaken to solicit user reactions to a set of Internet maps, to test whether professional geographers and non-geographers judge Internet maps differently, and to identify key issues in cartography related to the Internet. The study concentrates on Internet maps for tourism and travel. The paper does not offer definitive conclusions, but instead attempts to raise questions and methodological issues, and to stimulate debate.

Keywords: Maps, Internet, Map Design, User Survey, De-construction, Tourism

$\mathrm{T}$ he Internet, also known as the "Information Highway," "Infobahn," or the minimalist "Iway," offers new challenges and possibilities for the representation and communication of information, including cartographic information. Although the current focus on the Internet may exaggerate its true potential, it is undeniable that the Internet has already begun to have an impact on society - at least on that portion that owns a computer. Inevitably, the Internet will continue to establish itself as a source of information, and the map will be a part of it all.

Maps and the Internet would seem to be a perfect match: a new technology with which to display maps accessed by an audience numbering in the millions. Studying maps on the Internet is a logical next step in a research agenda where cartographers have studied maps in a variety of media: in newspapers and on television, (Gilmartin 1985, Balchin 1985), in magazines (Monmonier 1989), on journal covers (Paul 1990), and even on postage stamps (Davis 1985). Along this line, a pilot project was initiated to investigate Internet maps. This research was undertaken to fulfill the requirements of an undergraduate fourth year special reading course in advanced cartography. The ideas behind the pilot project were to pose and address methodological issues, to ask potential Internet users to critique the design elements and communication quality of a set of Internet maps, and to approach the survey with a post-modernist perspective as laid out by its two chief proponents, J. B. Harley (1990) and D. Wood (1993).

To achieve these goals and also gather general comments on the design and quality of Internet maps, a user survey was conducted of two different groups of potential Internet map users. A distinction was made between academic/professional geographers and non-geographers, hypothesizing that these two groups view Internet maps differently because of the former's professional familiarity with maps and their training as critical thinkers.

The study added a post-modernist perspective in order to learn how participants of a map survey would respond to the use of non-traditional cartographic measures, and to determine whether it would be possible to stimulate, as Harley put it, "a closer and deeper reading of the cartographic text" $(1989$, p. 8$)$ on the Internet. This is not to down-play the importance of traditional design and graphic elements in evaluating maps,
Mark Harrower

C. Peter Keller

Diana Hocking

Department of Geography and

Spatial Sciences Laboratories

University of Victoria

Victoria, $B C$

Canada V8W3P5

pkeller@office.geog.uvic.ca

\section{INTRODUCTION}

". . . the Internet will continue

to establish itself as a source of information, and the map will

be a part of it all." 
METHODOLOGY

The Internet "does not support a structured, objective or exhaustive search for Internet maps."

"Interactive maps were not included in the survey because they are not strictly 'standalone' documents." but to seek de-constructionist thoughts from the Internet map critics so that alternative methods of cartographic critique could be explored.

In summary, this paper reports on a survey designed

- to solicit user reaction to the quality and design of a set of Internet maps;

- to evaluate whether map users would consciously or sub-consciously de-construct Internet maps; and

- to test whether professional geographers and non-geographers view Internet maps differently.

The first part of this paper discusses the design of the survey and the reactions of the participants to a selection of Internet maps. This is followed by a more general discussion of cartography and the Internet, identifying key issues for the cyber-cartographer.

Three methodological issues had to be addressed:

- how to select a set of Internet maps to include in the survey;

- whom to survey; and

- how best to design and conduct the survey.

\section{Map Selection}

The Internet is a very large network that is difficult, if not impossible to search systematically. It was decided, therefore, to simplify the search by focusing on tourism and travel maps, assuming that there would be sufficient variety within this group to facilitate an interesting comparison. An advantage of the hypertext organization of information on the Internet is that it supports unstructured, personal freedom-of-information navigation. The corollary is that it does not support a structured, objective or exhaustive search for Internet maps. Thus, an informal and unstructured method was used to collect the Internet maps. The search started with a previously visited homepage that was known to be geared towards tourism and travel and from there followed links to other tourism and travel related homepages. If a dead-end was encountered, the steps would be retraced until another path could be explored. Internet maps relating to tourism and travel were selected if they had the correct theme, and did not have to rely upon additional text or graphics for explanation. Maps had to be designed as 'stand-alone' documents to ensure that they would be assessed fairly; even good maps can fail when they are taken out of context.

An initial sample of 18 maps was reduced to a set of ten maps. The emphasis in selection was on variety of quality and style with no conscious effort being made to select only the best or worst maps. Interactive maps were not included in the survey because they are not strictly 'stand-alone' documents and can not be reproduced outside of the hypertext environment. This was unfortunate because, although rare, interactive travel maps are some of the most exciting maps on the Internet.

One of the unanswered research questions identified in the pilot project was how to bring objectivity and structure into such a search and selection process. The cartographic research community will have to address this issue, and decide what is and what is not acceptable. It will be interesting to learn how other researchers will handle this methodological question of representative sampling of Internet maps, and how the research community will respond. 


\section{Selecting Survey Participants}

Because this was an undergraduate assignment to be completed within a limited time frame, and given the lack of financial support for the study, a relatively small sample of both professional geographers and non-geographers were surveyed. In the end, sixteen geographers and fourteen nongeographers participated in the survey. Members of the geography group were restricted to faculty, professional staff, and graduate students of the Geography Department at the University of Victoria, British Columbia. Non-geographers were defined as anyone without a college or university degree in geography, and without any formal training in cartography. This sample was drawn from the community in Victoria, British Columbia. Both groups were 'samples of convenience' although all efforts were made to avoid age, gender and educational bias, especially among nongeographers. The age of participants ranged from 21 to 63 years. There was an unavoidable gender bias towards males in the group of professional geographers owing to the present demographics of the Geography Department at the University of Victoria.

\section{Survey Design}

The ten Internet maps were arranged as a slide show presentation on a computer screen. The order in which the maps were presented was fixed in the same order as listed in Table 1 and shown at http:/ /

geography.geog.uvic.ca/dept/internet_maps. Participants controlled the

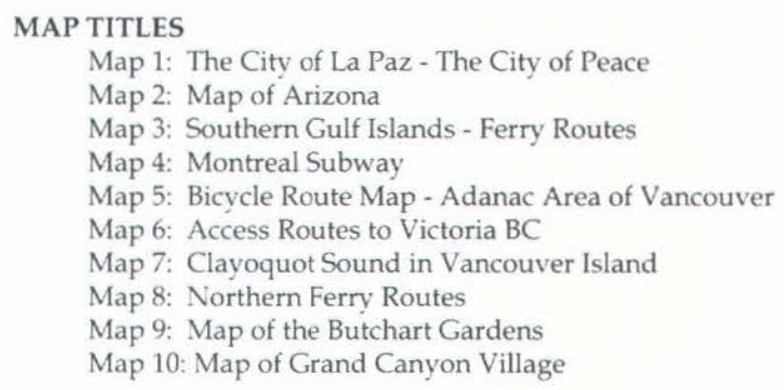

For additional information see http://geography.geog.uvic.ca/dept/internet_maps.

Table 1.

rate at which the slide show advanced. They were instructed to cycle through the slides and examine each map before commencing the map evaluation. The maps were displayed at their original size with titles included. In situations where there was no title on the original map, a title or reference was added. This title or reference led to the map from the hypertext link that activated the map.

A semantic differential approach was employed to record individuals' reactions to the maps. Survey respondents were given the set of questions listed in Figure 1. They were asked to rate their answers on a series of bipolar adjective scales using five word pairs: accurate-inaccurate, biasedunbiased, ethical-unethical, powerful-weak, convincing-unconvincing. No additional information was offered concerning these word pairs. Such a technique of recording individuals' reactions has been employed with
". . . sixteen geographers and fourteen non-geographers participated in the survey."
"They were asked to rate their answers on a series of bi-polar adjective scales using five word pairs: accurate-inaccurate, biased-unbiased, ethical-unethical, powerful-weak, convincingunconvincing." 
SURVEY RESULTS

"The similarity between the responses of the geographers and non-geographers was surprising." success in previous studies of maps (Taylor 1974, Dent 1975) and has been found to be sensitive to perceived differences between thematic maps (Gilmartin 1978).

Participants also were asked to record what they thought was the purpose of the map. An optional comments section was added to give participants an opportunity to record additional remarks or impressions. There was no time limit for completing the questionnaire, but most people required from 20 to 30 minutes to finish the survey, although some spent up to 1 hour.

MAP TITLE:

THE PURPOSE OF THE MAP IS:

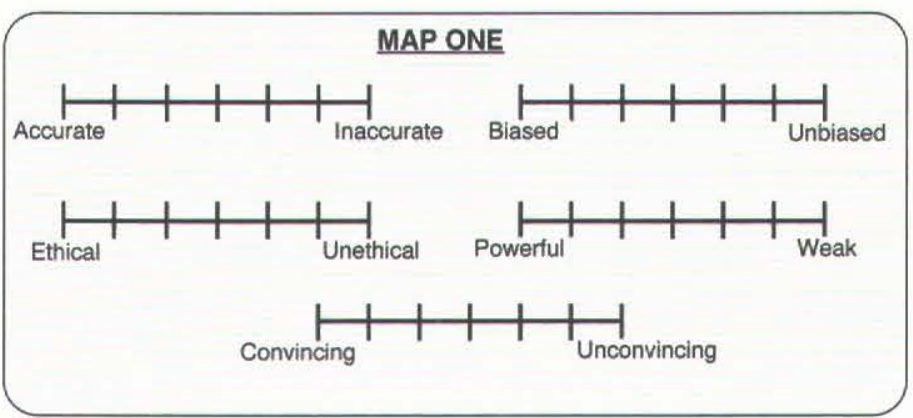

COMMENTS:

Figure 1.

Tables $2 a$ and $2 b$ show the summaries of the scores from the five bi-polar questions for the ten maps viewed by the professional geographers and non-geographers respectively. Shown are both mean and modal scores which were derived from the 7-point word-pair continuum. Potential scores for each word-pair ranged from a minimum of 1 (i.e."accurate") to a maximum of 7 (i.e. "inaccurate"). Respondents were told to leave the questions blank if they felt that a word did not apply to a given map. Table 3 shows the number of times the respondents chose this option. Tables 4 and 5 identify the maps that scored best and worst respectively using the five bi-polar questions and differentiating between professional geographers and non-geographers. Readers are encouraged to view the ten Internet maps when studying the results. Respondents' answers to 'map purpose' and their 'general comments' will be reported throughout the discussion below.

Both groups produced very similar mean and modal scores for each map. The similarity between the responses of the geographers and nongeographers was surprising. A Wilcoxon signed-ranks test was applied to the 50 word pairs ( 10 maps, 5 word pairs each) to test for between-group difference. Of the 50 group scores, only two (Map 6, "biased-unbiased", and Map 7, "powerful-weak") were found to be significantly different at the $95 \%$ confidence level. This represents 5 percent of our total, which could occur by chance. It is concluded, therefore, that the two groups did not score the maps differently. 
Table 2n. Geographers

\begin{tabular}{|c|l|l|l|l|l|}
\hline MAP & $\begin{array}{l}\text { Accurate - } \\
\text { Inaccurate }\end{array}$ & $\begin{array}{l}\text { Biased - } \\
\text { Unbiased }\end{array}$ & $\begin{array}{l}\text { Ethical - } \\
\text { Unethical }\end{array}$ & $\begin{array}{l}\text { Powerful - } \\
\text { Weak }\end{array}$ & $\begin{array}{l}\text { Convincing - } \\
\text { Unconvincing }\end{array}$ \\
\hline & Mean (Mode) & Mean (Mode) & Mean (Mode) & Mean (Mode) & Mean (Mode) \\
\hline 1 & $2.9(2)$ & $4.7(4)$ & $2.5(2)$ & $4.0(5)$ & $3.6(3)$ \\
2 & $3.2(2)$ & $3.8(2,3)$ & $3.0(4)$ & $3.5(2,3)$ & $2.9(2,3)$ \\
3 & $4.3(3)$ & $3.3(2)$ & $3.2(2)$ & $4.2(3)$ & $3.6(2,3)$ \\
4 & $2.5(1,2,4)$ & $3.9(3)$ & $2.2(2)$ & $2.1(1)$ & $2.0(1)$ \\
5 & $4.1(6)$ & $2.8(2)$ & $3.3(2,4)$ & $5.3(6)$ & $4.3(6)$ \\
6 & $4.5(5)$ & $3.8(3,4)$ & $3.6(4)$ & $5.3(5)$ & $4.8(4,6)$ \\
7 & $4.4(3,6)$ & $4.1(4)$ & $3.7(4)$ & $4.5(5)$ & $4.1(2,5)$ \\
8 & $4.4(6)$ & $3.6(3)$ & $3.5(2,4)$ & $4.9(5)$ & $4.4(5)$ \\
9 & $3.3(2,4)$ & $3.6(4)$ & $2.6(2)$ & $3.9(3)$ & $3.2(3)$ \\
10 & $2.7(3)$ & $3.7(3,4)$ & $2.8(2)$ & $2.9(4)$ & $3.4(3,4)$ \\
\hline
\end{tabular}

Table 2b. Non-Geographers

\begin{tabular}{|c|l|l|l|l|l|}
\hline MAP & $\begin{array}{l}\text { Accurate - } \\
\text { Inaccurate }\end{array}$ & $\begin{array}{l}\text { Biased - } \\
\text { Unbiased }\end{array}$ & $\begin{array}{l}\text { Ethical - } \\
\text { Unethical }\end{array}$ & $\begin{array}{l}\text { Powerful - } \\
\text { Weak }\end{array}$ & $\begin{array}{l}\text { Convincing - } \\
\text { Unconvincing }\end{array}$ \\
\hline & Mean (Mode) & Mean (Mode) & Mean (Mode) & Mean (Mode) & Mean (Mode) \\
\hline 1 & $2.5(2)$ & $4.6(4)$ & 3.1 (Blank) & $4.2(4)$ & $4.0(5)$ \\
2 & $2.9(2)$ & $2.3(1)$ & $3.5(4)$ & $2.9(2)$ & $2.6(2)$ \\
3 & $4.5(3)$ & $3.6(2,4,6)$ & $3.5(4)$ & $3.9(2)$ & $3.8(3)$ \\
4 & $2.7(1)$ & $3.8(2)$ & $1.8(4)$ & $2.0(1)$ & $1.5(1)$ \\
5 & $2.8(2)$ & $2.5(2)$ & 3.3 (Blank) & $4.1(2,6)$ & $4.5(4,6)$ \\
6 & $4.4(4)$ & $2.7(2)$ & $3.6(4)$ & $5.9(6)$ & $4.9(6)$ \\
7 & $3.9(3,4)$ & $3.2(2)$ & 3.3 (Blank) & $2.9(1,2)$ & $3.0(2)$ \\
8 & $3.9(4)$ & $2.8(2)$ & $4.0(4)$ & $4.4(6)$ & $4.6(4)$ \\
9 & $3.1(2,4)$ & $3.7(4)$ & 3.2 (Blank) & $3.4(3)$ & $2.8(2,3)$ \\
10 & $3.1(2)$ & $2.8(2,4)$ & $3.8(4)$ & $2.8(2)$ & $2.9(3)$ \\
\hline
\end{tabular}

Notes: 1. Multiple modes indicated.

2. Mode score of blank indicates that the most popular answer was to leave the continuum blank.

\begin{tabular}{|c|c|c|}
\hline Word Pair & $\begin{array}{l}\text { Geographers } \\
\text { (160 responses) }\end{array}$ & $\begin{array}{l}\text { Non-Geographers } \\
\text { (140 responses) }\end{array}$ \\
\hline Accurate - Inaccurate & $4 \quad(2.5 \%)$ & $0 \quad(0 \%)$ \\
\hline Biased - Lnbiased & $12(7.5 \%)$ & $16(11.4 \%)$ \\
\hline Ethical - Unethical & $28(17.5 \%)$ & $41(29.3 \%)$ \\
\hline Power - Weak & $1(0.6 \%)$ & $1 \quad(0.7 \%)$ \\
\hline Convincing - Unconvincing & $0 \quad(0 \%)$ & $3 \quad(2.1 \%)$ \\
\hline
\end{tabular}

Table 3. Number of Blank Responses

Tables 4 and 5 show that geographers and non-geographers generally agree upon what were the best and worst maps in each category, especially when combining best and worst maps with their next highest or lowest score. There appeared to be no explanation as to why the Montreal subway map (Map 4) scored consistently high. It is a map that abstracts geographic space and highly simplifies the geography of a transportation system, yet respondents perceived it as highly accurate, most ethical, most powerful and most convincing. The maps of access routes to Victoria (Map 6 and Map 8) consistently received the lowest scores. Comments revealed that respondents generally disliked both maps for the same reasons. Both geographers and non-geographers criticized the maps for lacking legends, titles, north arrows and scales. Illegibility of text and excessive spatial distortions were other common complaints.

"Both geographers and nongeographers criticized the maps for lacking legends, titles, north arrows and scales." 


\begin{tabular}{|c|c|c|}
\hline & Geographers & Non-Geographers \\
\hline Most Accurate & Map 4 (mean 2.5) & Map 1 (mean 2.5) \\
\hline Runner-up & Map 10 (mean 2.7) & Map 4 (mean 2.7) \\
\hline Least Biased & Map 1 (mean 4.7) & Map 1 (mean 4.6) \\
\hline Runner-up & Map 7 (mean 4.1) & Map 4 (mean 3.8) \\
\hline Most Ethical & Map 4 (mean 2.2) & Map 4 (mean 1.8) \\
\hline Runner-up & Map 1 (mean 2.5) & Map 1 (mean 3.8) \\
\hline Most Powerful & Map 4 (mean 2.1) & Map 4 (mean 2.0) \\
\hline Runner-up & Map 10 (mean 2.9) & Map 10 (mean 2.8) \\
\hline Most Convincing & Map 4 (mean 2.0) & Map 4 (mean 1.5) \\
\hline Runner-up & Map 2 (mean 2.9) & Map 2 (mean 2.6) \\
\hline \multicolumn{3}{|c|}{ Table 4. The "Good" Maps } \\
\hline & Geographers & Non-Geographers \\
\hline Least Accurate & Map 6 (mean 4.5) & Map 3 (mean 4.5) \\
\hline Runner-up & Map $7 / 8$ (mean 4.4) & Map 6 (mean 4.4) \\
\hline Most Biased & Map 5 (mean 2.8) & Map 2 (mean 2.3) \\
\hline Runner-up & Map 3 (mean 3.3) & Map 5 (mean 2.5) \\
\hline Least Ethical & Map 7 (mean 3.7) & Map 8 (mean 4.0) \\
\hline Runner-up & Map 6 (mean 3.6) & Map 10 (mean 3.8) \\
\hline Least Powerful & Map 5/6 (mean 5.3) & Map 6 (mean 5.9) \\
\hline Runner-up & Map 8(mean 4.9) & Map 8 (mean 4.4) \\
\hline Least Convincing & Map 6 (mean 4.8) & Map 6 (mean 4.9) \\
\hline Runner-up & Map 8 (mean 4.4) & Map 8 (mean 4.6) \\
\hline
\end{tabular}

Table 5. The "Bad" Maps

Table 3 reveals that both professional geographers and non-geographers had difficulty evaluating bias in the maps, and commenting on possible violation of ethics. Respondents indicated that the word pairs 'biased-unbiased' and 'ethical-unethical' were considered least applicable to the maps. This may mean that map readers are not familiar with or trained to evaluate maps in terms of bias and ethics, whereas the concepts of accuracy and power (effectiveness) of communication are familiar.

Professional cartographers accept that all maps distort and are biased

". . map readers are not familiar with or trained to evaluate maps in terms of bias and ethics..." because they show only a very selective portion of a three dimensional reality in two dimensions (Monmonier 1991). Cartographers know that maps are abstracted, authored pieces of communication. Nonetheless, it would appear that many of the survey participants from both groups expressed uncertainty or confusion at the idea of assessing the ethics or bias of a map. Future research is required to determine whether the blank responses are an indication that the viewers felt the Internet maps were unbiased or unethical, or whether they saw the concepts of bias and ethics as an inappropriate measure of maps. The latter seems the more probable explanation.

MacEachren (1995) points out that maps with well-chosen colors and that include neat, easy to read text and symbols subtly suggest to the viewer that they are truthful; that these maps will inspire confidence. He agrees with Wood (1993) that the 'best' maps are neat, trim and efficient, never flashy. Indeed, for a map to work, it must connote veracity (Wood 
1993). The results of the survey supports these observations. The maps that were rated the most accurate, ethical and convincing were also the maps that looked the most professional by our standards. Comments for these maps included "easy to read", "nice choice of colors", and "effective". It is suspected that most cartographers would agree with our respondents' selections of the best maps. An interesting point is that these maps were also assumed to be the most accurate and the most ethical of the sample; perhaps because they were the best looking.

Tourist maps are full of "cartographic silences." They do not necessarily show where the competition is located, what you will see as you drive to the attraction (e.g. clear-cuts from logging), or the presence of a foulsmelling pulp mill located across the river. Nonetheless, only once did a survey respondent suggest that a map was "not telling the whole story". This respondent knew that one of the maps did not show all of the possible routes to a destination and concluded, therefore, that the map communicated a bias toward the major local commercial carrier, in this case British Columbia Ferries.

The two most disliked maps in the survey were those depicting ferry routes to and from Victoria. Because these maps were thematically and graphically similar, they were included in the survey to check the consistency of participant evaluations. Participants criticized the excessive spatial distortions and inaccuracies in these maps which were subsequently characterized as inaccurate, unreliable, sloppy, amateurish, and devoid of integrity. It is of interest to observe that, by comparison, the map of the Montreal subway system received very favorable comments and was rated by both groups as the most ethical, powerful and convincing map of the survey. The extreme spatial distortions of the subway map did not appear to bother viewers although it was evident that subway routes are not straight lines and that the stations are not evenly spaced. Perhaps the geographic distortions inherent in the Montreal subway map were considered acceptable because they created a simpler, easier to understand map, or because subways travel underground in a world that is less familiar - a world where it is not possible to look at scenery out the window - so there is not need to care about geographic accuracy. Some of the map viewers did note that relative locations are more important on subway maps than actual location. But, there is a certain inconsistency in map evaluation given that ferry route maps were not extended the same cartographic license.

Analysis of respondents' answers to the "purpose of the map" revealed that there was great similarity in response, both between and within groups. Responses were typically variations on or an elaboration of the titles provided. This led to the conclusion that Internet map viewers took the maps at "face value." Participants did not attempt to read a deeper purpose or ascribe an ulterior motive to the maps.

After testing to determine whether professional geographers and nongeographers view and critique Internet maps differently, the results reported in Tables 1 through 5 were not able to prove significant differences. Professional geographers did not produce a deeper and more thorough analysis of Internet maps.

An attempt was also made to explore whether map users would consciously or sub-consciously de-construct Internet maps. If as post-modernists believe, maps are imbued with layers of meaning, hidden agendas and political overtones, these were not apparent to the survey participants through either direct or indirect questioning. De-construction is, at heart, the search for alternative meanings (Harley 1989). No alternative meanings were identified by the respondents in the set of Internet maps. Pre-
"Tourist maps are full of 'cartographic silences'."

"Professional geographers did not produce a deeper and more thorough analysis of Internet maps." 
INTERNET MAP TYPES

"Maps designed and drafted specifically to suit their place on the Internet were only rarely encountered." liminary conclusions, therefore, are threefold. First, map readers are naive and simplistic in their use of maps, and lack the critical thinking skills necessary to de-construct Internet maps. Second, the survey methodology failed to elicit what it set out to measure because of methodological flaws. Third, map de-construction is an interesting academic exercise with little application in the real world. Future research is required to definitively verify any of the three possible conclusions. Of special interest would be to see how those who have taken a cartography course dealing with postmodernism and de-constructionism would interpret maps.

This study has given us some insights into the maps that are available through the Internet. The following are some specific comments on tourism and travel maps on the Internet, as well as general comments on the types of maps that are available through the Internet.

Searches revealed a general shortage of tourism and travel maps. Numerous homepages for tourist destinations and attractions had no maps of any kind. Given that these pages exist presumably to inform, raise awareness or entice people to visit, the lack of even simple locator maps is mysterious. A case in point is the official homepage for Disneyland. This homepage was rich with information about the park, all geared to entice and inform potential visitors. However, the hypertext link entitled "How to get to Disneyland" presents the viewer with four paragraphs of text describing the highway system of southern California. There are no highway or locator maps on this homepage. This oversight is typical and emphasizes the need for more travel and tourism maps on the Internet, as well as opportunities for cartographers to supply those maps.

A more general investigation of Internet maps revealed that most maps have one of two origins. Generally, they are scanned paper maps but occasionally they are digital maps created for purposes other than display on the Internet. A third hybrid class of maps has evolved. These maps were originally a conventional scanned paper map, but were subsequently digitally edited or customized. Maps designed and drafted specifically to suit their place on the Internet were only rarely encountered.

Where maps designed specifically for the Internet were found, they exploited the new technology to its fullest. The Internet had interactive maps revealing hypertext linked layers of information that encouraged the viewer to explore the study area at various scales and from different thematic angles. Some of these maps were found to provide links to associate attribute information and supporting text (for example, an accompanying news story) activated by a simple click of the mouse. Some of these maps were intuitive, intelligent and easy to use. Some verged on pieces of art.

An example of a web-savvy design is the interactive Penn State campus map (http://www.psu.edu:80/psu/UniversityPark/campusmap.html). This site actually is a "virtual tour" of the campus that provides visitors with zoom-capable maps (activated by clicking on the map itself) which reveal increasingly detailed information. At the largest scale, photographs accompany the maps, enhancing the "tour" experience. This site was easy to navigate and decidedly user-friendly.

The "real-time" sea-surface temperature maps produced by the Space Science and Engineering Center (SSEC) incorporate another exciting new possibility of Internet cartography, i.e., real-time maps (http:// www.ssec.wisc.edu/data/sst/latest_sst.gif). Rapid and extensive dissemination of information has long been recognized as a key attribute of the Internet. The Web gives cartographers the opportunity to produce an up-to-date map and have it used later that day by people around the world. Currently, weather maps and satellite images are the most com- 
mon type of "real-time" spatial data available on the Web, but there are other tantalizing possibilities such as traffic-flow maps of metropolitan areas, and maps which display environmental events such as oil spills and forest fires. Realizing the full potential of real-time cartography will require the development of automated GIS expert systems that continuously receive, process, and publish information over the Internet.

Animated maps are another recent cartographic development taking advantage of the Internet. Currently, some of the best examples are produced by the National Geophysical Data Center at http:/ / www.ngdc. noaa.gov $/ \mathrm{mgg} /$ image/images.html. These maps are fascinating to view although awkward to download given their file size. They are extremely data-rich documents that are usually measured in megabytes, rather than the kilobytes of static maps. This data-volume problem will remain a concern until data transmission and network connection speeds increase.

The rare innovative map taking full advantage of the latest advances in 3-dimensional and virtual reality 'plug-ins' (software designed to enhance your web browser) demonstrated the potential that the Internet has to offer cartography. The new hypermedia technology can free the cartographer from conventional design constraints. Of course, all these technological design opportunities do not guarantee a better map - the general principles of good design do not change, even when the technology does.

Unfortunately, it must be reported that while great design opportunities exist, the majority of today's Internet maps are low quality. Scanned paper maps do not translate well to the new medium. Major problems include image quality degradation, warping from improper scanning, coarse scanning resolutions, and over-reduction which renders many maps unreadable. Commonly, when serif and italicized fonts are used on paper maps, they become blurred in the transfer to a digital format.

In examining Internet maps today, it is clear that scanning is a reductive process, and that there is a fundamental difference between paper and digital maps, especially digital maps on the Internet. The digital medium dictates that digital maps must be significantly less complex to remain legible at $72 \mathrm{dpi}$ (the standard resolution of computer monitors). Furthermore, Internet maps must be small documents given that the average user is downloading them with a 14.4 baud or, at best, 28.8 baud modem. Few Internet users are willing to wait the 10 minutes it takes to download some of the largest maps included in the sample. On the Internet, size (digital storage) of image is very important. Internet map designers will need to realize that an Internet map must be accessible in a few seconds in order to be effective. Until connection speeds increase, a $100 \mathrm{k}$ document is perhaps a realistic ceiling.

A possible solution to the problem of excessive document size is the use of layered maps. By incorporating a few basic elements of GIS technology, the cyber-cartographer could provide "assembled" maps consisting of a set of files, each significantly less complex than an entire map, and hence, smaller digital documents. For example, the traditional topographic map in its paper form is a data-rich item that serves a variety of map users well. Unfortunately, it does not translate well to the Web because it is too complex. The digital equivalent of the topographic map, therefore, will have to be simpler. If the digital version could be delivered as layers, it could then be customized and users would get exactly what they wanted.

Another problem with many of the maps on the Internet is that they can not be displayed in their entirety on a standard computer screen. The image must be scrolled in order to view it all and to find relevant informa-
". . the general principles of good design do not change, even when the technology does."

INTERNET MAP QUALITY

\author{
"A possible solution to the \\ problem of excessive document \\ size is the use of layered maps."
}


"The challenge is to merge the best design aspects of traditional cartography with the opportunities offered by the new medium."

\section{SUMMARY AND CONCLUSION}

tion, including title, legend, etc. These maps are difficult to read, frustrating and confusing. In addition, many of the travel maps failed to offer basic cartographic information (north arrows, scales, legends), leaving viewers bewildered or frustrated. Maps should entice, not confuse. The general poor design and many fundamental cartographic mistakes in design led us to two conclusions. First, most cartographic products presented on the Internet are produced or edited by people with little or no formal cartographic training. Secondly, the new computer medium has removed some of the traditional design controls; that is, the graphic artist and/or cartographer can no longer dictate how their product is displayed.

Traditionally, the cartographer had control over map presentation: the size of the map, type of paper, the printing technique, etc.. These controls do not exist in cyberspace. Those designing graphics for the Internet must accept that their work will be viewed on a wide range of monitors with varying quality, size and color capabilities. The same digital map will look vastly different from monitor to monitor. Internet cartographers must consider how their product, created on a top-of-the-line 21 inch color monitor, will look if downloaded by a user with a 15 inch monochrome monitor.

A possible solution to this dilemma is, of course, to provide more than one version of the same map. Cartographers could offer the choice between a deluxe version of a map intended for the user with a fast connection and high-end equipment, and a regular version that caters to the 'worst-case scenario' user (i.e. a black and white monitor, slow modem). The cyber-cartographer, as a courtesy to the user, should always include a thumbnail sketch of the map (i.e. 5 kilobytes) that would allow the user to get a sense of image complexity and whether it is worthwhile to download the image. The latter could simply be included along with the map title.

Although the quality of paper maps has not yet been duplicated on the computer screen via the Internet, digital advances should eventually help achieve this level of quality. Creating facsimiles of the paper map on the computer screen, however, should not be the driving force behind Internet cartography. The Internet medium offers opportunities for innovation in map design, including map animation, variable scale and variable resolution maps, multi-media, map interaction, and customized mapping. The challenge is to merge the best design aspects of traditional cartography with the opportunities offered by the new medium.

Internet cartography is in its infancy. The Internet creates both new challenges and new opportunities for the cartographer. Little is known about what users want from and expect of Internet maps. This pilot survey raises some methodological issues that need to be resolved and also showed that most of today's Internet maps were not designed by trained cartographers. Cartographers may have designed the original paper maps from which the majority of Internet maps were scanned - but it is unlikely that they controlled the final appearance of their maps on the computer screens. As Keller and Wood (1996) pointed out, cartographers have two choices when faced with technological change. Cartographers can choose to watch passively as others, presumably graphic artists and computer specialists, continue to shape the future of cartography on the Internet, or cartographers can choose to become active players themselves.

The challenge is for cartographers to realize the full potential of the new information medium, to be willing to abandon efforts to reproduce paper maps on the computer screen, and to take over active leadership in 
the emerging cyber-cartography. In the process, cartographers should not be concerned solely with the technology. They should remember the words of Brian Harley, Mark Monmonier, Denis Wood and others who explain that all maps are potentially imbued with layers of meaning, hidden agendas and political overtones, and that there is no such thing as a single homogeneous group of map users. When embracing the new technologies and the new opportunities, cartographers should understand how the Internet may change representation and communication of space and place, and what inadvertent messages may be delivered to their audiences.

There clearly is a need for both, more maps and better maps on the Internet, and considerable research to be done. The study reported here is simply an attempt to raise some questions and methodological issues, and to stimulate debate.

Balchin, W. G. V. (1985) "Media map watch", The Geographical Magazine, 57(8), p.408-409.

Davis, B. (1985) "Maps on postage stamps as propaganda", The Cartographic Journal, 22, p. 125-130.

Dent, B. D. (1975) "Communication aspects of value-by-area cartograms", The American Cartographer, 2(2), p. 154-168.

Gilmartin, P. P. (1985) "The design of journalistic maps: purposes, parameters and prospects", Cartographica, 22(4), p. 1.

Gilmartin, P. P. (1978) "Evaluation of thematic maps using the semantic differential test", American Cartographer, 5(2), p. 133-139.

Harley, J. B. (1990) "Cartography, ethics, and social theory", Cartographica, $27(2)$, p. $1-23$.

Harley, J. B. (1989) "Deconstructing the map", Cartographica, 26(2), p. 1-20.

Keller, C. P. and C. H. Wood (1996) "Advances in Cartographic Design". Chapter 20 (p. 277-285) in Cartographic Design: Theoretical and Practical Perspectives. C. H. Wood and C. P. Keller, eds., John Wiley and Sons,

Chichester, U.K..

MacEachren, A. M. (1995) How Maps Work. Guilford Press, New York.

Monmonier, M. (1991) How to Lie with Maps. University of Chicago Press, Chicago.

Monmonier, M. (1989) Maps with the News. University of Chicago Press, Chicago.

Paul, B. K. (1990) "Maps of journal covers", Geographica, 75(1), p. 52-57. Taylor, R. M. (1974) “An application of subjective scaling techniques to map evaluation", Cartographic Journal, 11(3), p. 246-252.

Wood, D. (1993) "The power of maps", Scientific American (May 1993), p. $88-93$. 